\title{
Simulations of Effects of Nanophase Iron Space Weather Products on Lunar Regolith Reflectance Spectra
}

\section{Escobar-Cerezo, J.}

2018-01-20

Escobar-Cerezo , J , Penttilä, A , Kohout , T , Munoz , O , Moreno , F \& Muinonen , K 2018 , ' Simulations of Effects of Nanophase Iron Space Weather Products on Lunar Regolith Reflectance Spectra ' , Astrophysical Journal , vol. 853 , no. 1 , 71 . https://doi.org/10.3847/1538-4357/aaa24d

http://hdl.handle.net/10138/232531

https://doi.org/10.3847/1538-4357/aaa24d

unspecified

publishedVersion

Downloaded from Helda, University of Helsinki institutional repository.

This is an electronic reprint of the original article.

This reprint may differ from the original in pagination and typographic detail.

Please cite the original version. 


\title{
Simulations of Effects of Nanophase Iron Space Weather Products on Lunar Regolith Reflectance Spectra
}

\author{
J. Escobar-Cerezo ${ }^{1}$ (i) , A. Penttilä ${ }^{2}$, T. Kohout ${ }^{2,3}$, O. Muñoz ${ }^{1}$ (iD, F. Moreno ${ }^{1}$ (i), and K. Muinonen ${ }^{2,4}$ \\ ${ }^{1}$ Instituto de Astrofísica de Andalucía, CSIC, Glorieta de la Astronomía s/n, E-18008 Granada, Spain \\ ${ }_{3}^{2}$ Department of Physics, P.O. Box 64, FI-00014 University of Helsinki, Finland \\ ${ }^{3}$ Institute of Geology, The Czech Academy of Sciences, Prague, Czech Republic \\ ${ }^{4}$ National Land Survey of Finland, Finnish Geospatial Research Institute, P.O. Box 84, FI-00521 Helsinki, Finland \\ Received 2017 May 29; revised 2017 December 14; accepted 2017 December 15; published 2018 January 24
}

\begin{abstract}
Lunar soil spectra differ from pulverized lunar rocks spectra by reddening and darkening effects, and shallower absorption bands. These effects have been described in the past as a consequence of space weathering. In this work, we focus on the effects of nanophase iron $\left(\mathrm{npFe}^{0}\right)$ inclusions on the experimental reflectance spectra of lunar regolith particles. The reflectance spectra are computed using SIRIS3, a code that combines ray optics with radiative-transfer modeling to simulate light scattering by different types of scatterers. The imaginary part of the refractive index as a function of wavelength of immature lunar soil is derived by comparison with the measured spectra of the corresponding material. Furthermore, the effect of adding nanophase iron inclusions on the reflectance spectra is studied. The computed spectra qualitatively reproduce the observed effects of space weathered lunar regolith.
\end{abstract}

Key words: comets: general - methods: numerical - minor planets, asteroids: general - planets and satellites: surfaces - scattering

\section{Introduction}

Space weathering has been extensively studied and characterized through the last decades. It affects any atmosphereless solar system body. The main interests of space weathering effects over the reflectance spectra are centered in the Moon (Pieters et al. 1993), asteroids (Clark et al. 2002; Brunetto et al. 2015), and planets (e.g., Mercury, Domingue et al. 2014). In the present work, we focus on computer simulations of the effects of space weathering on lunar regolith.

The first lunar soil samples were brought to Earth in 1969 by the Apollo 11 mission. A typical lunar soil spectrum shows an increase of reflectance with wavelength (reddening) as well as weak absorption bands at 1 and $2 \mu \mathrm{m}$. The bands present at 1 and $2 \mu \mathrm{m}$ are due to $\mathrm{Fe}^{2+}$ in silicates. Olivine has three overlapping bands at $1 \mu \mathrm{m}$, while pyroxenes have one at $1 \mu \mathrm{m}$ and another at $2 \mu \mathrm{m}$ (Burns 1970; Cloutis \& Gaffey 1991). The absorption band at $1 \mu \mathrm{m}$ is consistent with those present in silicate type particles, and the weak $2 \mu \mathrm{m}$ band is typical of pyroxenes.

On the lunar surface, it was observed that freshly exposed material from meteoroid impacts are brighter than the surrounding regolith deposits. Spectra from pulverized lunar rocks from Apollo missions were studied to compare with the regolith (Pieters et al. 1993). This pulverized material has a higher albedo than the soil gathered in the same spot. Moreover, the absorption bands are more pronounced and the spectrum has a flatter slope. In comparison, lunar soils have weaker absorption bands, larger slope of the spectrum at longer wavelengths (reddening) and lower values of reflectance for all wavelength ranges (darkening). When the lunar soils were analyzed to explain these differences, abundant dark glass particles were discovered, mainly in the agglutinates. In a first approach, a vitrification origin of lunar rocks by meteorite impact was suggested, with the consequent darkening (Conel \& Nash 1970). A lunar rock sample was melted, and the obtained material had optical features consistent with those observed in the lunar soil, i.e., dark and reddish material with shallower absorption bands. However, subsequent attempts to reproduce these results were unfruitful as they differ from what was expected (Cassidy \& Hapke 1975; Hapke et al. 1975). The new experimentally vitrified material had high albedo, flat spectra (no reddening), and strong absorption bands. The differences between these two experiments were the environmental conditions. As pointed out by Wells \& Hapke (1977), the initial experiments were conducted in a regular atmosphere (Conel \& Nash 1970), whereas these new experiments were made in a vacuum chamber, along with different crucible material. This produced oxidizing conditions for the first experiment, generating ferric oxides or $\mathrm{Fe}^{3+}$, which have strong absorption bands similar to those found in lunar regolith. Vitrification was then discarded as a possible explanation for these spectrum features.

Two experiments were performed by Gold et al. (1970) and Hapke et al. (1970) to test the hypothesis of a surface origin of the space weathering effects. Lunar samples were treated with a solution of $20 \%$ hydrochloric acid. $\mathrm{HCl}$ washed the surface of the grains without affecting their sizes. The washing of the surfaces produced an increase in the albedos, deeper absorption bands, and a decrease of the spectrum slope. As the lunar rocks are milled, fresh surfaces are exposed, and the effect of the weathered surfaces decreases.

Several numerical models has been developed in the past to mimic the effects of space weathering on the reflectance spectra (see, e.g., Hapke 2001; Lucey \& Riner 2011; Trang et al. 2017). These models are based on radiative-transfer models and Maxwell-Garnett theory. In this work, we use the SIRIS3 code as described in Muinonen et al. (2009). The code combines the ray optics and radiative-transfer treatments to compute light scattering by irregular particles much larger than the wavelength of the incident light. In geometric optics, there are no interference effects among the fields scattered by particles in 
the system and thus the scattered electromagnetic field can be considered incoherent, significantly simplifying the computations. The ray optics code also accounts for internal medium of diffuse scatterers mimicking inclusions inside the particle. Different versions of SIRIS have been previously used for reproducing the experimental scattering matrices of clouds of irregular mineral dust particles (Muinonen et al. 1996; Nousiainen et al. 2011; Escobar-Cerezo et al. 2017). A recent work report on the performance of SIRIS for reproducing the spectral properties of meteorites in the ultraviolet, visible, and nearinfrared ranges (Martikainen et al. 2018).

In the present paper, we test the performance of SIRIS3 for modeling the effect of the nanophase iron $\left(\mathrm{nFFe}^{0}\right)$ space weathering product on the reflectance spectra of lunar regolith. In particular, we retrieve information about the space weathering nanophase iron $\mathrm{npFe}^{0}$ volume fraction by comparing between experimental and computed spectra. Furthermore, we derive the imaginary part of the refractive index $k_{\text {host }}$ of an immature lunar sample in the wavelength range from 300 to $2600 \mathrm{~nm}$. This $k_{\text {host }}$ is derived by fitting the experimental reflectance spectrum of an immature soil sample to mimic a non-weathered host particle. From there, four models are computed with four different volume fractions of $\mathrm{npFe}$ inclusions inside the host particle. We use two experimental spectra from the NASA RELAB (Reflectance Experiment Laboratory) facility at Brown University for comparison and as a starting model. These experimental spectra correspond to two lunar soil spectra from samples named 12030,56 and 12001,853, which are presented in Section 3.

\section{Space Weathering}

Space weathering is produced by galactic and solar cosmic rays, sputtering from solar wind particles and bombardment by micrometeorites. Laboratory experiments suggest a timescale of $10^{4}-10^{6}$ years for inducing the space weathering effects observed on the reflectance spectra from S-type near-Earth asteroids (Strazzulla et al. 2005). However, other experiments suggest that this time can be as short as $10^{3}-10^{4}$ years in asteroids (Brunetto et al. 2014). This exposure age of space weathered soils can be characterized approximately by the $\mathrm{I}_{s} / \mathrm{FeO}$ index, known as the maturity index, where $\mathrm{I}_{s}$ is the characteristic ferromagnetic resonance from submicroscopic (single-domain) iron and $\mathrm{FeO}$ is the weight percent of $\mathrm{FeO}$ in the soil. As the exposure of the soil increases, the singledomain iron amount increases, and thus the value of $\mathrm{I}_{s} / \mathrm{FeO}$ is higher.

In the case of the lunar regolith the $\mathrm{FeO}$ content in mare soils is, on average, at least three times greater than in highland soils implying larger values of $\mathrm{I}_{s}$ in the former one (Pieters et al. 2000; Noble et al. 2001). Small nanophase iron $\left(\mathrm{npFe}^{0}\right)$ is formed by space weathering processes near the surface of lunar grains (Keller \& McKay 1997). The amount of $n p F e^{0}$ depends on several factors, as the initial availability of $\mathrm{Fe}^{2+}$ in the regolith source, the micrometeorites kinetic energy and the frequency of impact, the ion radiation produced by the Sun, and the exposure time to this environment (Pieters et al. 2000). In some cases, several layers of $\mathrm{nFFe}^{0}$ are observed, suggesting different episodes of weathering processes (Pieters et al. 2000). Dedicated laboratory experiments have been performed through the years to produce space weathering products on diverse substrates through different methods: laser irradiation (e.g., Brunetto et al. 2005), ion bombardment (e.g., Marchi et al. 2005), microwave irradiation (e.g., Tang et al. 2012), or chemical reduction, such as in Noble et al. (2007) in silica gels or Kohout et al. (2014) in olivine. In the case of Noble et al. (2007), the silica gel particles contain pores ranging between 2.6 and $50 \mathrm{~nm}$. A treatment with ferric nitrate solutions, drying and posterior calcination produced iron oxide deposits in those pores. The samples were then introduced in a furnace under reducing conditions to obtain several sizes of $\mathrm{npFe}^{0}$ deposits (from 5 to $200 \mathrm{~nm}$ ). Those samples were then studied to check the effect of the $n p F e^{0}$ size on the reflectance spectra. The Kohout et al. (2014) experiment worked in a similar way, but in this case the naturally present iron in the olivine was the source to produce $n p F e^{0}$. Through two heating processes they could produce different amounts and sizes of $\mathrm{npFe}^{0}$ deposits. Both experiments reproduced the space weathering effects, namely, darkening, reddening and subdued absorption bands on the reflectance spectra. Moreover, they studied the effects of quantity and size of these products on the reflectance spectra indicating that lunar soil continuum is better simulated with $n \mathrm{pFe}^{0}$ spheres with sizes ranging from 15 to $25 \mathrm{~nm}$ (Noble et al. 2007).

Another space weathering product is the so-called BrittPieters microphase. It consists of $\mathrm{Fe}^{0}$ particles larger than $33 \mathrm{~nm}$ in the regolith (Britt \& Pieters 1994). The nanophase iron $\left(n p F e^{0}\right)$ seems to be the principal mechanism for producing darkening, reddening and subdued absorption bands on the reflectance spectra of lunar regolith (Pieters et al. 1993), while the Britt-Pieters microphase only produces darkening (Pieters \& Noble 2016). In this work, we focus on the effects of $n p \mathrm{Fe}^{0}$ inclusions on the lunar reflectance spectra.

\section{Experimental Spectra of Lunar Samples}

In this section, we present two experimental spectra corresponding to two lunar soil samples with different maturity indexes, namely, samples 12030 (McKay et al. 1972) and 12001 (Morris 1978), collected in the Apollo 12 mission. Physical characterization of these samples can be found in Morris (1980) and Taylor et al. (2001). These spectra have been selected as a representative of an immature spectrum and a mature spectrum, respectively. We focus on the measured reflectance spectra of subsamples consisting of grains in the size range from 20 to $45 \mu \mathrm{m}$. In particular, subsample 12030,56 is composed of particles ranging from 30 to $37 \mu \mathrm{m}$ with an $\mathrm{I}_{s} / \mathrm{FeO}$ value of 12 i.e., it can be considered as an immature soil sample. Subsample 12001,853 consists of dust particles in the $20-45 \mu \mathrm{m}$ size range with an $\mathrm{I}_{s} / \mathrm{FeO}$ value of 51 (submature).

Their measured reflectance spectra are presented in Figure 1 (top panel). We notice the absorption band weakening as $\mathrm{I}_{s} / \mathrm{FeO}$ increases. Also the darkening of the spectra is perceived. To facilitate the slope comparison of the reddening effect, the measurements are normalized at the wavelength of $550 \mathrm{~nm}$ in the bottom panel of this figure. Here we clearly see the reddening effect as the maturity index increases. Moreover, the absorption band dampening is easily observed.

\section{Modeling Approach}

In this work, we use a numerical code based on ray optics with diffuse and specular interactions, the so-called SIRIS3 code. The method is thoroughly described by Muinonen et al. (2009), so that we only give a brief description. Diffraction and 

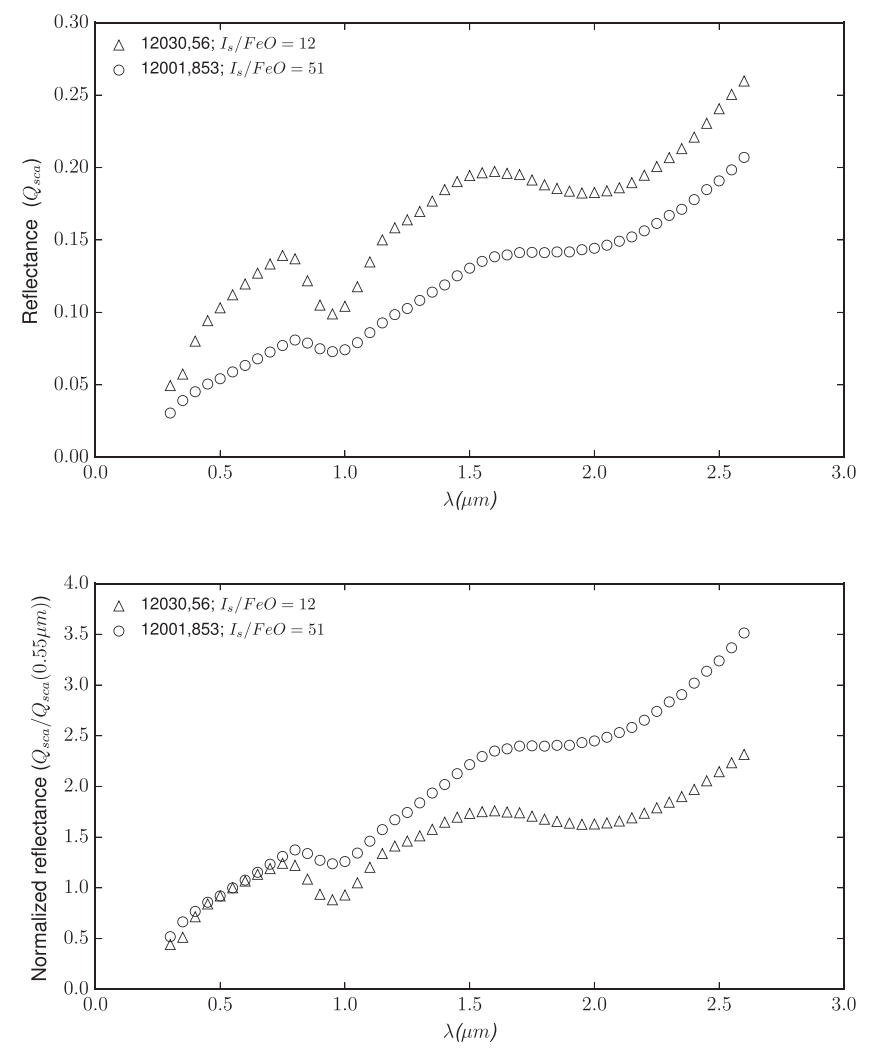

Figure 1. Reflectance spectra of two lunar regolith samples. Sample 12030,56 is an immature regolith $\left(\mathrm{I}_{s} / \mathrm{FeO}=12\right)$, and sample 12001,853 is submature $\left(\mathrm{I}_{s} / \mathrm{FeO}=51\right)$. These data are available at NASA RELAB database.

geometric optics are treated separately, although in this work diffraction is not considered. Moreover, it takes into account internal and/or external diffuse scattering media. The diffuse scatterers can constitute an internal medium distributed uniformly across the particle interior (DIM, for diffuse internal medium). DIM is defined by the volume fraction $\rho$ and by its scattering properties, i.e., single-scattering albedo $\varpi$ and extinction efficiency $Q_{\text {ext }}$. The scatterers are placed in an isotropic medium, which we name the host particle, and they represent small-scale features that affect the scattering. The scattering behavior of these inclusions can be simulated inside SIRIS3 using either double Henyey-Greenstein functions, customized input matrices, or Rayleigh scattering. The latter case is the one selected in this work due to the small size of the inhomogeneities $\left(\mathrm{npFe}{ }^{0}\right.$ smaller than $20 \mathrm{~nm}$ ).

\subsection{Particle Shapes}

The host particle shape is generated as a Gaussian random sphere (GRS), as detailed in Muinonen et al. (2009). In spherical coordinates, they are described by a radius vector that is the exponential of a Gaussian random process:

$$
\begin{gathered}
r(\vartheta, \phi) \boldsymbol{e}_{r}=\frac{a \exp [s(\vartheta, \phi)]}{\sqrt{1+\sigma^{2}}} \boldsymbol{e}_{r} \\
s(\vartheta, \phi)=\sum_{l=0}^{\infty} \sum_{m=-l}^{l} s_{l m} Y_{l m}(\vartheta, \phi) \\
s_{l,-m}=(-1)^{m} s_{l m}^{*},
\end{gathered}
$$

where $s(\vartheta, \phi)$ is the logarithmic radial distance, $Y_{l m}$ are orthonormal spherical harmonics, and $s_{l m}$ are Gaussian random variables with zero means. The parameters $a$ and $\sigma$ are the mean and relative standard deviation. The standard deviation of the Gaussian random variables $s_{l m}$ follow the covariance function $\Sigma_{s}$, which is given by a series of Legendre polynomials $P_{l}$. The degree $l$ of these polynomials range from 0 to $\infty$, but in the code the series is truncated by $l_{\min }$ and $l_{\max }$. The greater the value of $l_{\min }$, the spikier the particle will be, reducing its sphericity accordingly.

The code parameters for the GRS are the mean radius of the host particle $r, \sigma$ which describes the relative standard deviation from the sphere's surface, $\nu$ which sets the power law of the covariance function, $l_{\min }$ and $l_{\max }$ to fix the coefficients of the Legendre polynomials and the correlation angle for autocorrelation. The values of these parameters (except the radius $r$ ) are fixed for all the simulations presented in this work: $\sigma=0.2, \nu=3.3, l_{\min }=2$, and $l_{\max }=11$. These values are in agreement with the shape distribution of a silicate sample collected in the Sahara desert (Libya) presented in Muñoz et al. (2007), which is considered to be a reasonable standard irregular particle.

\subsection{Simulation Methodology}

The procedure consists of three steps. First, we compute the imaginary part of the refractive index, $k_{\text {host }}$, of the nonweathered host particle. Then, a first simulation of a weathered particle is performed by adding iron inclusions $\left(\mathrm{nFe}^{0}\right)$ to the non-weathered host particle. The result of this simulation is equivalent to the single-scattering behavior of irregular space weathered particles. To fully simulate the reflectance spectra of an ensemble of regolith particles, a radiative-transfer simulation is computed by using the results of the single-scattering particles acting as inclusions within a $10 \mathrm{~cm}$ radius vacuum sphere.

The simulations cover the wavelength range from 300 to $2600 \mathrm{~nm}$ with a resolution of $20 \mathrm{~nm}$. In all computed cases, the particle size is fixed to $33.5 \mu \mathrm{m}$ as an average value representative of the studied samples. One of the multiple outputs of the code is the scattering efficiency, $Q_{\text {sca }}$, which is defined as the ratio of the scattering cross section $\sigma_{\text {sca }}$ to the geometrical cross section $\pi a^{2}$, where $a$ is the host particle radius. The dependence of $Q_{\text {sca }}$ versus the wavelength $\lambda$ conforms to the reflectance spectrum.

The wavelength dependence of $k_{\text {host }}$ is derived by SIRIS3 as follows: we perform six sets of simulations with $k_{\text {host }}$ ranging between $8 \cdot 10^{-5}$ and $3 \cdot 10^{-2}$ to cover a wide range of values. In each case, $k_{\text {host }}$ is constant along the wavelength range. The real part of the refractive index is fixed to 1.67 at all computed wavelengths (Goguen et al. 2010). A reflectance spectrum is computed by SIRIS3 for each $k_{\text {host }}$. The reflectance spectrum of the immature sample 12030,56 is fitted by combining all six of the simulations (Figure 2). The $k_{\text {host }}(\lambda)$ values obtained by this fitting as presented in Figure 3, are then used to simulate the host particle.

The scattering matrix and single-scattering albedos for $33.5 \mu \mathrm{m}$ lunar grains with $\mathrm{npFe}^{0}$ inclusions are computed using GRSs in SIRIS3. The scattering properties of the inclusions are computed using Rayleigh approximation, due to their small size compared to the wavelength $(15-25 \mathrm{~nm})$. In the Rayleigh approximation, the scattering efficiency $Q_{\text {sca }}$ and the absorption efficiency $Q_{\mathrm{abs}}$ of a particle with a size 


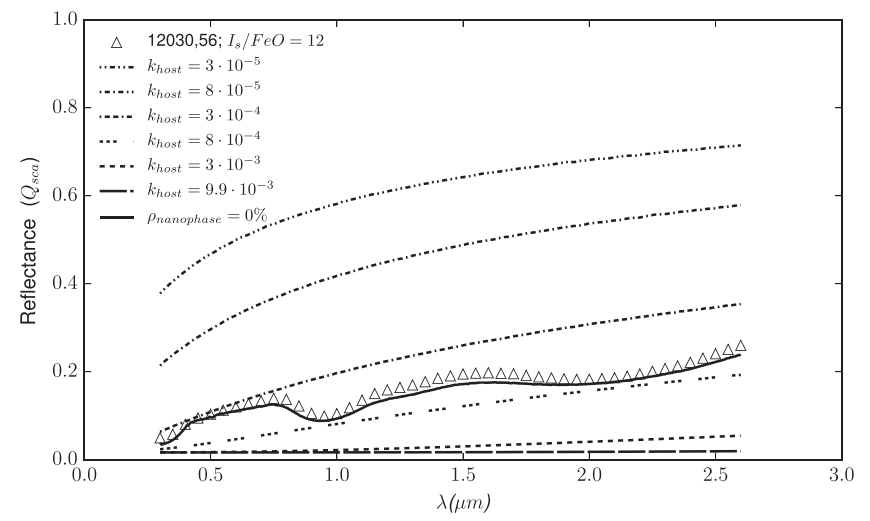

Figure 2. Fitting model for non-weathered host particle. The triangles correspond to the experimental spectrum for sample 12030,56, which acts as a model. The dashed lines are the computed spectra when using a constant $k_{\text {host }}$. The solid line is the spectra resulting when fitting the model with the computations.

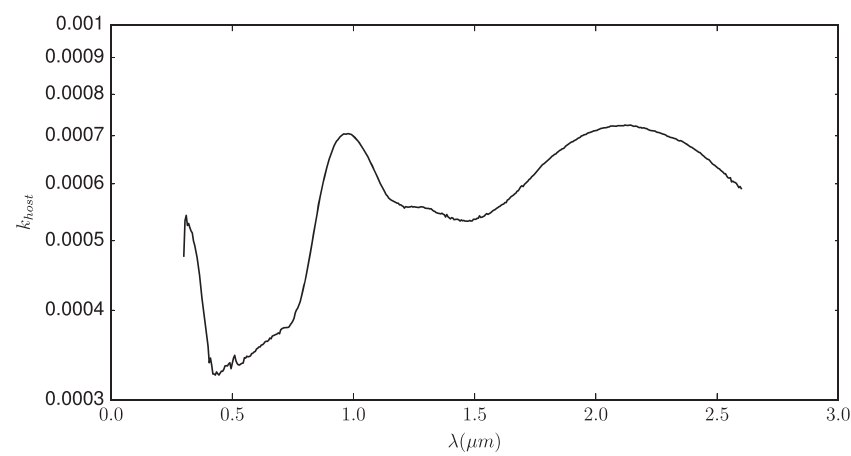

Figure 3. Modeled imaginary part of the refractive index $\left(k_{\text {host }}\right)$ for the nonweathered particle. The experimental spectrum of 12030,56 is used as a nonweathered sample.

parameter $x=\frac{2 \pi a}{\lambda}$ are given by

$$
Q_{\text {sca }}=\frac{8}{3} x^{4}\left|\frac{m_{\text {iron }}^{2}-1}{m_{\text {iron }}^{2}+2}\right|^{2}, \quad Q_{\mathrm{abs}}=4 x \operatorname{Im}\left(\frac{m_{\text {iron }}^{2}-1}{m_{\text {iron }}^{2}+2}\right),
$$

where $m_{\text {iron }}$ is the relative complex refractive index of the iron particle in the host particle matrix. We obtain the complex refractive index for iron from Cahill et al. (2012). The actual inputs for the SIRIS3 code, the single-scattering albedo $\varpi_{\text {iron, }}$, and volume fraction of inclusions, $\rho_{\text {nanophase }}$ in the host matrix, are derived as

$$
\varpi_{\text {iron }}=\frac{Q_{\text {sca }}}{Q_{\text {sca }}+Q_{\text {abs }}} \text { and } \rho_{\text {nanophase }}=\frac{4 a}{3 \ell\left(Q_{\text {sca }}+Q_{\text {abs }}\right)},
$$

where $\ell$ is the mean-free path in the host matrix and $a$ is the radius of each $n p \mathrm{Fe}^{0}$ inclusion, set to $8.25 \mathrm{~nm}$. Each inclusion has a size of $16.5 \mathrm{~nm}$, half of the $33 \mathrm{~nm}$ size established as the upper limit to consider it nanophase iron.

The obtained scattering properties for the inclusions are then used in SIRIS3 so that the $\mathrm{npFe}^{0}$ particles act as diffuse scatterers inside a $33.5 \mu \mathrm{m}$ non-weathered host lunar grain. The inclusions are assumed to be uniformly distributed inside the grain. The amount of inclusions is controlled by means of the volume fraction parameter $\rho_{\text {nanophase }}$.

In order to model the reflectance spectrum of space weathered lunar regolith, the computed scattering matrices
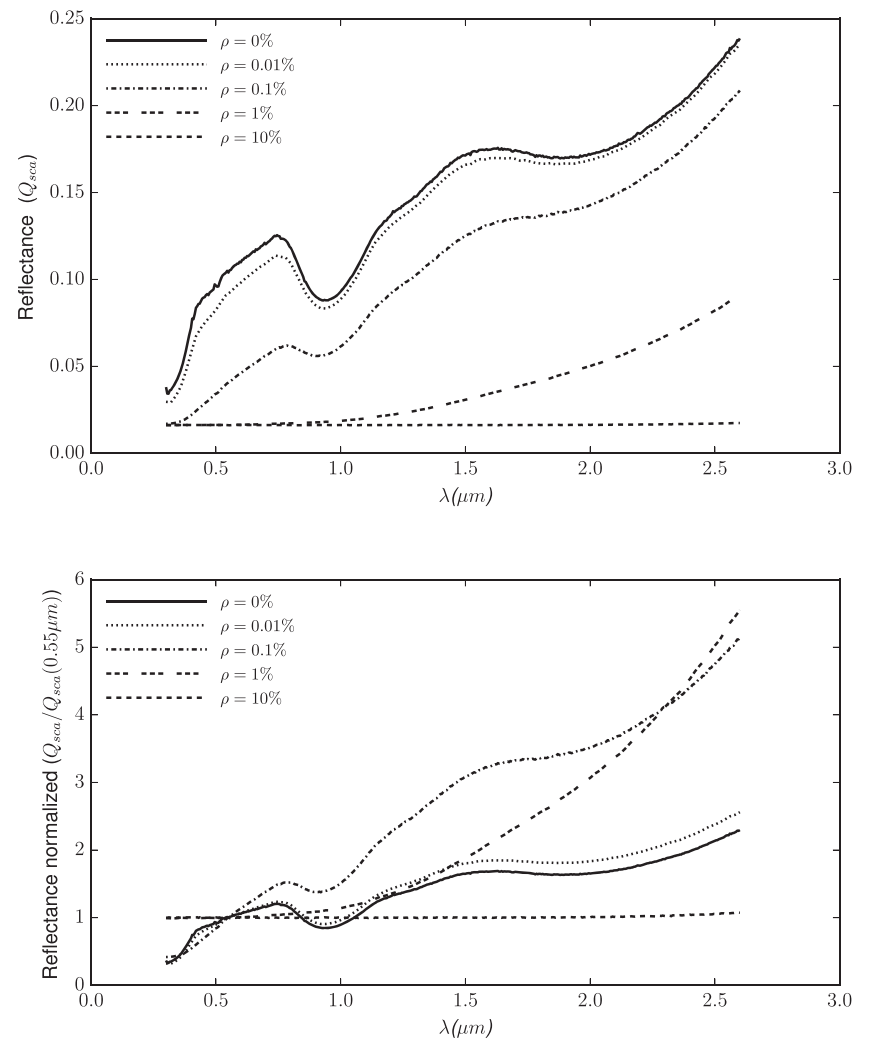

Figure 4. Computed reflectance spectra for five different volume fractions $\left(\rho_{\text {nanophase }}=0 \%, 0.01 \%, 0.1 \%, 1 \%\right.$, and $\left.10 \%\right)$. The $\rho_{\text {nanophase }}=0 \%$ curve is the fitted result of experimental spectrum soil 12030,56. The top panel corresponds to the original simulation data. The bottom panel shows the same spectra normalized to unity at $550 \mathrm{~nm}$.

and single-scattering albedos of the weathered particles are used in the code so that $33.5 \mu \mathrm{m}$ lunar grains with internal inclusions act as diffuse scatterers inside a vacuum volume of a radius of $10 \mathrm{~cm}$. The volume fraction of lunar grains in the vacuum space was set to $\rho_{\text {grains }}=15 \%$.

\section{Results}

In Figure 3, we present the retrieved imaginary part of the refractive index $\left(k_{\text {host }}\right)$ as a function of wavelength for nonweathered lunar regolith. The results are based on the bestfitted reflectance spectrum for the immature 12030,56 lunar sample displayed in Figure 2.

In Figure 4, we present the dependence of the computed reflectance spectra on different values of the $\mathrm{npFe}^{0}$ volume fraction, $\rho_{\text {nanophase, }}$ which is varied in the range $0.01 \%$ to $10 \%$. As is shown (Figure 4, top panel), even in the case of a very small amount of inclusions (as low as $\rho_{\text {nanophase }}=0.01 \%$ ), the darkening and dampening of absorption bands are clearly seen in the computed spectra. In contrast, when $\rho_{\text {nanophase }}=1 \%$, the spectrum becomes unrealistic: all structure has disappeared and the spectrum becomes almost featureless at visible wavelengths. At $\rho_{\text {nanophase }}=10 \%$, the spectrum corresponds to a completely black surface. In the bottom panel of the same figure, the reddening can be easily observed between $\rho_{\text {nanophase }}=0 \%$ and $\rho_{\text {nanophase }}=0.1 \%$. For values $\rho_{\text {nanophase }} \geqslant 1 \%$, the spectra start to fade in the visible range. Hereafter, we focus only in those $\rho_{\text {nanophase }} \geqslant 1 \%$ results.

In Figure 5, we present a comparison between the experimental spectrum for sample 12001,853 and spectra 

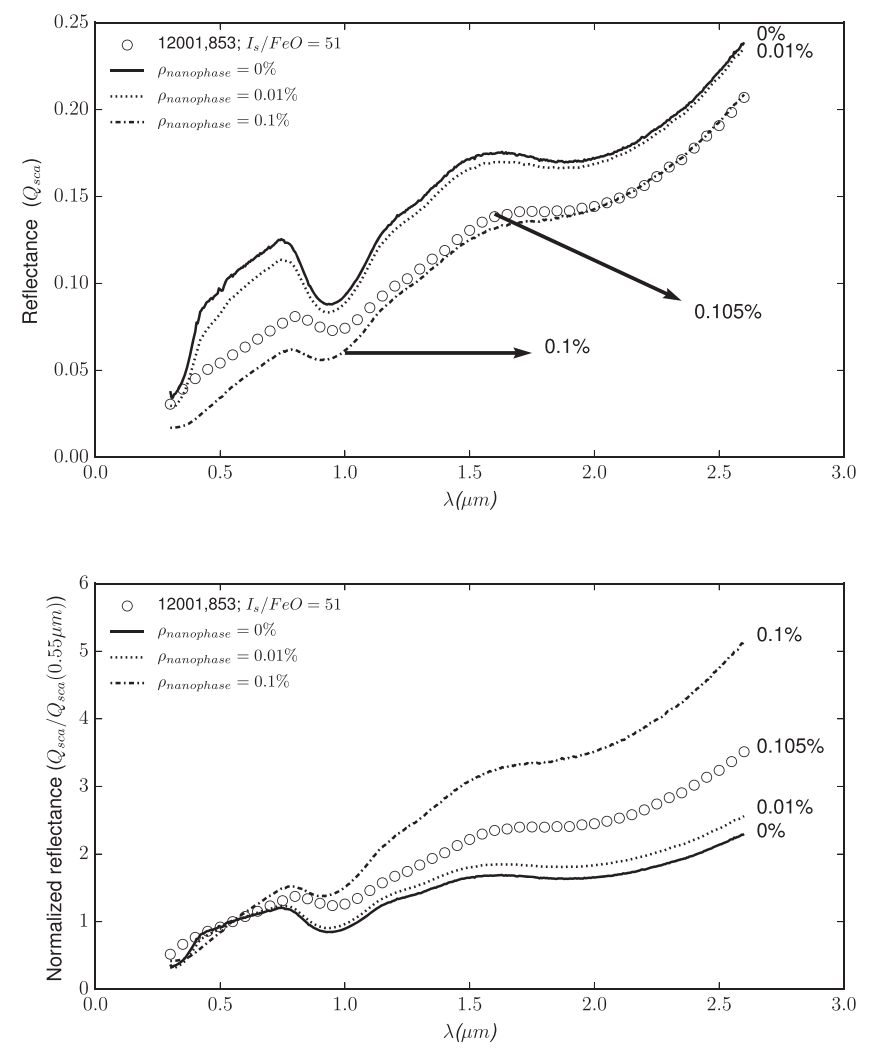

Figure 5. Top panel shows reflectance spectra comparison between the experimental lunar regolith spectrum for sample 12001,853 and three computed reflectance spectra with space weathering inclusions (volume fractions $\rho_{\text {nanophase }}=0 \%, 0.01 \%$, and $0.1 \%$ ). The numbers next to each spectrum are the correspondent volume fraction (computed for the sample and used as input for the simulations). Bottom panel shows the same spectra normalized to unity at $550 \mathrm{~nm}$.

simulations for various values of $\rho_{\text {nanophase }}$ In the bottom panel (normalized data), the experimental spectra are located between the $\rho_{\text {nanophase }}=0.01 \%$ and $0.1 \%$ simulation spectra.

\section{Discussion}

As shown in Figure 4, SIRIS3 code qualitatively reproduces the measured effects on the reflectance spectra as the amount of $n p \mathrm{Fe}^{0}$ is increased. The reflectance spectrum darkens, the slope becomes steeper and the absorption bands tend to vanish. In Figure 5, we find that the experimental spectrum for sample 12001,853 is located between the simulated spectra for values $\rho_{\text {nanophase }}=0.01 \%$ and $\rho_{\text {nanophase }}=0.1 \%$.

To our knowledge, there are no published data of the volume fraction of nanophase iron inclusions for the samples studied in the present work. To compare the results of our simulations with measured properties of the samples, we use two equations to transform the $\mathrm{FeO} \mathrm{wt} \%$ (weight percent) and the maturity index $\left(\mathrm{I}_{s} / \mathrm{FeO}\right)$ into $\mathrm{npFe}^{0}$ volume fraction.

First, we use the empirical equation from Morris (1980) to obtain the $\mathrm{Fe}^{0} / \mathrm{FeO}$ ratio corresponding to $\mathrm{Fe}^{0}$ with sizes from 4 to $33 \mathrm{~nm}$ as is the case in our simulations:

$$
\frac{\mathrm{Fe}^{0}}{\mathrm{FeO}}=\left[(3.20 \pm .08) \times 10^{-4}\right] \frac{\mathrm{I}_{s}}{\mathrm{FeO}}+(1 \pm 5) \times 10^{-4}
$$

Taylor et al. (2001) provide $\mathrm{FeO}$ wt\% values of 17.6 and 16.9 for samples 12030,56 and 12001,853 , respectively, along with the maturity index of both samples. From the previous equation and those data, we obtain the values of $\mathrm{Fe}^{0}$ weight percent of $0.069 \pm 0.013$ for sample 12030,56, and $0.28 \pm 0.03$ for sample 12001,853. To transform from weight percent to volume fraction, we use the equation from Lucey \& Riner (2011):

$$
V_{\mathrm{Fe}}=\frac{M_{\mathrm{Fe}} \rho_{h}}{\rho_{\mathrm{Fe}}},
$$

where $M_{\mathrm{Fe}}$ is the $\mathrm{Fe}^{0} \mathrm{wt} \%, \rho_{h}$ is the host particle density, and $\rho_{\mathrm{Fe}}$ is the Fe density. As $\rho_{h}$, we use a mean value of $3 \mathrm{~g} \mathrm{~cm}^{-3}$ (Colwell et al. 2007), and for $\rho_{\mathrm{Fe}}$, we use $7.86 \mathrm{~g} \mathrm{~cm}^{-3}$. Using those values, Equation (7), and the results from the Equation (6) for the samples studied in this work, we obtain $\rho_{\text {nanophase }}=0.023 \%$ for sample 12030,56 and $\rho_{\text {nanophase }}=0.105 \%$ for sample 12001,853 .

As shown in Figure 5, the modeled spectrum is nearly consistent with the observed spectrum; however, the model has a slightly higher reflectance than the observed spectrum and it is not as red. The computed spectrum for $\rho_{\text {nanophase }}=0.1 \%$ is very similar to the measured spectrum for sample 12001,853 . In particular, it shows a nearly perfect fit to the experimental spectra in the wavelength range from 1.3 to $2.6 \mu \mathrm{m}$. Some differences with the experimental data are found in the $0.3-1.2 \mu \mathrm{m}$ wavelength range. Several sources of errors in the simulations might be taken into consideration as a possible explanation of the observed discrepancies at shorter wavelengths. As mentioned, in our computations, we assume the nanophase iron inclusions uniformly distributed throughout the host particle volume instead of locating the $\mathrm{npFe}^{0}$ on a thin layer close the surface of the grain. Another issue is the monodispersity of the size distribution of the iron inclusions in the host particle, which could be more realistically replicated by taking into account the Britt-Pieters microphase, but this requires a different simulation method. It would also be interesting to compute a size distribution of host particles to check whether the regolith spectrum is more accurately reproduced.

\section{Conclusions}

The SIRIS3 code qualitatively reproduces the effects of nanophase iron inclusions on the reflectance spectra of space weathered lunar soils. As a first step, a non-weathered reflectance spectrum is simulated. As a result, an imaginary refractive index model as a function of wavelength is obtained for immature lunar regolith. Once we have an immature regolith model, we reproduce the space weathering by assuming Rayleigh iron internal scatterers inside a large host particle. In the simulations, the increase of $n p F e^{0}$ volume fraction produces a darkening effect, a reddening of the spectra and weakening of the absorption bands. A saturation of the space weathering effects is found for $\rho_{\text {nanophase }} \geqslant 1 \%$, as the spectrum flattens with a very low reflectance.

A comparison between the experimental spectrum for lunar soil 12001,853 with computed spectra for $\rho_{\text {nanophase }}=0 \%$, $0.01 \%$, and $0.1 \%$ is made. When comparing simulated and experimental spectra, we find a good agreement between experimental and simulated $n p \mathrm{Fe}^{0}$ volume fractions. Still some discrepancies between experimental and computed spectra are found. This could be due to the fact that not all the parameters influencing the weathering effects could have been properly taken into account. Among other reasons, and owing to a limitation of the code, the nanophase iron has been assumed to 
be uniformly distributed inside the host particle instead of being located near the surface. Also, the internal inclusions and the host particles are simulated with a single size instead of a size distribution, which could be more realistic.

The overall results show that space weathering must be considered when computing the scattering of such susceptible samples as those found in airless bodies. The role of $n \mathrm{pFe}^{0}$ inclusions in simulations is essential to reproduce and characterize the reddening, darkening, and weakening of absorption bands in the reflectance spectra of these samples. Although this ray optic code with diffuse internal medium has demonstrated its ability to nearly reproduce the space weathering effects over visible to nearinfrared spectra, further improvements should be made. It would be necessary to implement the $\mathrm{npFe}^{0}$ layer depth and the possibility to mimic $\mathrm{npFe}^{0}$ size distributions, as well as other space weathering subproducts.

We highly appreciate the time and effort of the anonymous referee. Her/his constructive criticism and valuable comments have helped us to significantly improve the quality of this work. This work has been supported by the Plan Nacional de Astronomía y Astrofísica contracts AYA2015-67152-R and AYA2015-71975-REDT. Jesús Escobar-Cerezo, Antti Penttilä, and Karri Muinonen acknowledge the support from the ERC project No. 320773 "SAEMPL." Tomas Kohout is supported by the Academy of Finland project No. 285432, and by the Institute of Geology (Czech Academy of Sciences), supported by Ministry of Education, Youth and Sports project No. RVO67985831. Computational resources were provided by CSC-IT Centre for Science Ltd, Finland.

\section{ORCID iDs}

J. Escobar-Cerezo (1) https://orcid.org/0000-0003-0432-4443

O. Muñoz (i) https://orcid.org/0000-0002-5138-3932

F. Moreno (1) https://orcid.org/0000-0003-0670-356X

\section{References}

Britt, D. T., \& Pieters, C. M. 1994, GeCoA, 98, 3905

Brunetto, R., Lantz, C., Ledu, D., et al. 2014, Icar, 237, 278

Brunetto, R., Loeffler, M. J., Nesvorný, D., et al. 2015, in Asteroids IV, ed. P. Michel et al. (Tucson, AZ: Univ. Arizona Press), 597

Brunetto, R., Romano, F., Blanco, A., et al. 2005, Icar, 180, 546

Burns, R. G. 1970, AmMin, 55, 1608

Cahill, J. T. S., Blewett, D. T., Nguyen, N. V., et al. 2012, GeoRL, 39, L10204 Cassidy, W., \& Hapke, B. 1975, Icar, 25, 371

Clark, B. E., Hapke, B., Pieters, C., \& Britt, D. 2002, in Asteroids III, ed. W. F. Bottke, Jr. et al. (Tucson, AZ: Univ. Arizona Press), 585 Cloutis, E. A., \& Gaffey, M. J. 1991, JGR, 96, 22809

Colwell, J., Batiste, S., Horányi, M., et al. 2007, RvGeo, 45, RG2006

Conel, J., \& Nash, D. 1970, GeCAS, 1, 2013

Domingue, D. L., Chapman, C. R., Killen, R. M., et al. 2014, SSRv, 181, 121

Escobar-Cerezo, J., Palmer, C., Muñoz, O., et al. 2017, ApJ, 838, 74

Goguen, J., Stone, T., Kieffer, H., \& Buratti, B. 2010, Icar, 208, 548

Gold, T., Campbell, M., \& O'Leary, B. 1970, GeCAS, 1, 2149

Hapke, B. 2001, JGR, 106, 10039

Hapke, B., Cassidy, W., \& Wells, E. 1975, Moon, 13, 339

Hapke, B., Cohen, A., Cassidy, W., \& Wells, E. 1970, GeCAS, 1, 2199

Keller, L., \& McKay, D. 1997, GeCoA, 61, 2311

Kohout, T., Čuda, J., Filip, J., et al. 2014, Icar, 237, 75

Lucey, P. G., \& Riner, M. A. 2011, Icar, 212, 451

Marchi, S., Brunetto, R., Magrin, S., et al. 2005, A\&A, 443, 769

Martikainen, J., Penttilä, A., Gritsevich, M., et al. 2018, JQSRT, 208, 144

McKay, D. S., Heiken, G. H., Taylor, R. M., et al. 1972, LPSC, 3, 983

Morris, R. 1978, LPSC, 9, 760

Morris, R. 1980, LPSC, 11, 1697

Muinonen, K., Nousiainen, T., Fast, P., et al. 1996, JQSRT, 55, 577

Muinonen, K., Nousiainen, T., Lindqvist, H., et al. 2009, JQSRT, 110, 1628

Muñoz, O., Volten, H., Hovenier, J. W., et al. 2007, JGR, 112, D13215

Noble, S., Pieters, C., \& Keller, L. 2007, Icar, 192, 629

Noble, S., Pieters, C., Taylor, L., et al. 2001, M\&PS, 36, 31

Nousiainen, T., Muñoz, O., Lindqvist, H., et al. 2011, JQSRT, 112, 420

Pieters, C. M., Fischer, E. M., Rode, O., \& Basu, A. 1993, JGR, 98, 20817

Pieters, C. M., \& Noble, S. K. 2016, JGRE, 121, 1865

Pieters, C. M., Taylor, L. A., Noble, S. K., et al. 2000, M\&PS, 35, 1101

Strazzulla, G., Dotto, E., Binzel, R., et al. 2005, Icar, 174, 31

Tang, H., Wang, S., \& Li, X. 2012, P\&SS, 60, 322

Taylor, L. A., Pieters, C. M., Keller, L. P., et al. 2001, JGR, 106, 27985

Trang, D., Lucey, P. G., \& Izenberg, N. R. 2017, Icar, 293, 206

Wells, E., \& Hapke, B. 1977, Sci, 195, 977 